\title{
ESTIMATIVA DA ABUNDÂNCIA DE MINERAIS DO DEPÓSITO DE NÍQUEL LATERÍTICO DA MINA FAZENDA POR ESPECTRORRADIOMETRIA
}

\author{
OSMAR ABÍLIO DE CARVALHO JÚNIOR ${ }^{1}$, ÉDER DE SOUZA MARTINS ${ }^{2}$, ANA PAULA \\ FERREIRA DE CARVALHO ${ }^{3}$, PAULO ROBERTO MENESES $^{4}$, RENATO FONTES GUIMARÃES ${ }^{5}$ \\ \& FABRÍCIO BUENO DA FONSECA CARDOSO 6
}

\begin{abstract}
ESTIMATE OF MINERALABUNDANCE BY SPECTRORADIOMETRY IN THE MINA FAZENDA LATERITIC NICKEL DEPOSIT, BRAZIL The aim of the paper is to develop a method to estimate the relative abundance of pimelite in relation to goethite and asbolan present in the lateritic nickel deposit (Niquelândia - GO), from reflectance spectroscopy data. Spectral mixtures, of macroscopic and microscopic (intimate) type were analysed. The behavior of intimate mixture was analyzed by known amounts of the minerals and its respective measure in the laboratory. The macroscopic mixtures were made by mathematical simulation using linear functions. Both cases were employed index based on the $\mathrm{Mg}-\mathrm{OH}$ spectral feature present in the pimelite. Mixture index and the band depths were used for spectral analyses. The index values were adjusted with the known amounts found in the sample by linear and not linear regressions according to the case. Thus, extreme situations of mixtures had been established. The results show that the developed model is appropriate to estimate relative concentration of pimelite. This spectral analysis method has the advantage to obtain a remote mineralogical characterization of lateritic nickel horizon profile.
\end{abstract}

Keywords: Macroscopics Mixtures, Intimate Mixtures, Lateritic nickel, Pimelite.

Resumo O presente trabalho visa desenvolver um método para estimar a abundância de pimelita em relação a goethita e asbolana, presente no depósito supergênico de Niquelândia (GO), a partir de dados espectrorradiométricos. Foram avaliadas tanto as condições de mistura íntima como de mistura macroscópica. O comportamento da mistura íntima foi analisado por meio de quantidades conhecidas dos minerais e sua medição em laboratório. As misturas macroscópicas foram feitas por simulações matemáticas usando funções lineares. Em ambos os casos foram empregados índices baseados na feição espectral $\mathrm{Mg}-\mathrm{OH}$ presente na pimelita. Para a análise espectral utilizou-se a profundidade da banda e os coeficientes da regressão linear. Os valores provenientes dos índices foram ajustados com as quantidades verdadeiras encontradas na amostra por meio de regressões lineares e não lineares conforme o caso. Desta forma, foram estabelecidas situações extremas de misturas. O modelo desenvolvido mostrou-se adequado para a estimar a concentração relativa de pimelita. Este método de análise espectral apresenta a vantagem de obter uma caracterização mineralógica remota de horizontes de perfis de níquel laterítico.

Palavras-Chave: Mistura Macroscópica, Mistura Íntima, Níquel Laterítico, Pimelita.

\begin{abstract}
INTRODUÇÃO A adequação de métodos que utilizam a radiometria na faixa do visível e infra vermelho próximo, para estimar a abundância mineral no perfil laterítico, apresenta vantagem na rapidez da aquisição da informação. Devido à alta portabilidade dos espectrorradiômetros de campo pode-se realizar um acompanhamento mineralógico no próprio local de extração, reduzindo o tempo e priorizando amostras para análise em laboratório. Além disso, com o avanço da espectroscopia de imageamento pode-se ter um acompanhamento da distribuição mineral em planta das minas de céu aberto utilizando técnicas de processamento digital. No entanto, a estimativa de abundância a partir de dados de espectrorradiometria necessita de uma calibração que considere os tipos de misturas minerais.
\end{abstract}

A mistura espectral é proveniente da integração de dois ou mais componentes distintos que geram um comportamento espectral híbrido de seus integrantes. Desta forma, o espectro de uma mistura é uma combinação das reflectâncias dos membros puros ou finais presentes. Pode-se distinguir dois tipos de mistura com comportamentos distintos no cálculo de abundância: a mis- tura macroscópica e a mistura microscópica ou mistura íntima.

O objetivo deste artigo é o de empregar técnicas de espectrorradiometria para diferenciar e caracterizar a composição mineral dos perfis de intemperismo do depósito de níquel da mina Fazenda, Niquelândia (GO). O depósito é do tipo silicatado e difere de outros depósitos do mundo, onde o minério ocorre na zona oxidada, como nas Filipinas (Santos-Ynigo 1964), Venezuela (Jurkovic 1963), Austrália (Turner 1968) e África do Sul (Waal 1971).

O intemperismo das rochas ultramáficas de Niquelândia apresenta uma peculiar concentração do $\mathrm{Ni}$ em filossilicatos secundários. Essa característica é evidente pelos estudos petrográficos e químicos dos perfis de intemperismo das minas Jacuba, em Niquelândia, que apresenta em contexto similar à mina Fazenda (Colin et al. 1985 1990). A natureza do arcabouço geológico (intercalação de dunito e piroxenito) e do contexto geomorfológico (vales suspensos em piroxenitos balizados por colinas de dunitos) juntamente com as condições climáticas, são os principais condicionantes que se combinam para permitir a retenção do $\mathrm{Ni}$ nessa forma. Os silicatos secundários de níquel nos depósitos de

1 - INPE - Instituto Nacional de Pesquisas Espaciais - 12201-970 - São José dos Campos - SP, Brasil - osmar@ltid.inpe.br

2 - EMBRAPA Cerrados - Rodovia Brasília Fortaleza, km 18 Planaltina Distrito Federal. eder@cpac.embrapa.br

3 - Departamento de Ecologia - UnB - Campus Universitário Darcy Ribeiro, Asa Norte, 70910-900, Brasília, Distrito Federal. anapaula@unb.br

4 - Instituto de Geociências - UnB - Campus Universitário Darcy Ribeiro, Asa Norte, 70910-900, Brasília, Distrito Federal. pmeneses@unb.br

5 - Dep. Geografia - UnB - Campus Universitário Darcy Ribeiro, Asa Norte, 70910-900, Brasília, Distrito Federal. renatofg@solar.com.br

6 - CNPq - SEPN 509 Bloco A - Ed. Nazir I, 2 andar, COCTC - DF, Asa Norte, 70750-901, Brasília,DF - Brasil. fbueno@cnpq.br 
Niquelândia são provenientes do piroxênio e caracterizam-se por alta concentração de níquel contrapondo-se às outras mineralizações descritas no mundo, oriundas da olivina e serpentinita com mais baixo teor (Melfi et al. 1980, Oliveira \& Trescasses 1980). A distinção da fácies oxidada em relação à fácies silicatada é primordial para definir as porções do perfil com maiores concentrações do minério.

Na mina Fazenda, a principal esmectita portadora de níquel é a pimelita, que apresenta uma coloração esverdeada (Carvalho Júnior 2000). Em direção do topo observa-se a presença da Fe-saponita e goethita, que dão uma coloração marrom ao minério. A zona oxidada é formada predominantemente por goethita. No depósito é muito freqüente a presença de fendas preenchidas por asbolana (óxidos de Co-Mn-Ni).

O presente trabalho busca comparar metodologias para quantificação de pimelita por meio de dados espectrais em relação à goethita e asbolana na mina de Niquelândia (GO). Desta forma, busca-se estimar a abundância relativa de minerais silicatados em relação à fase oxidada importante para descrição do perfil de intemperismo e a concentração relativa de minério.

MISTURA MACROSCÓPICA Esse modelo considera que a probabilidade de um fóton ser espalhado entre os constituintes de uma mistura é dependente apenas da área de contribuição de cada espécime presente e não de um prévio espalhamento do fóton (Johnson et al. 1983). Este fato torna a reflectância espectral de uma determinada superfície uma combinação linear dos seus componentes, sendo o modelo denominado de tabuleiro de xadrez (checkerboard). A equação (1) expressa que o espectro da mistura $(E m)$ para um dado comprimento de onda $(\lambda)$ é obtido pela simples média ponderada entre os espectros (E) dos componentes presentes, sendo o valor do peso a fração da área do material na superfície da mistura (f).

$$
\operatorname{Em}(\lambda)=f E(\lambda)
$$

O comportamento da mistura macroscópica é ilustrado na figura 1 , pela simulação de uma mistura espectral com $50 \%$ de vegetação e de $50 \%$ de solo. Este último, por sua vez, é subdividido em três porções iguais dos minerais caulinita, gibsita e goethita.

Esse modelo pode ser aplicado em superfícies com partículas grandes e rugosas onde relativamente pouca luz é espalhada entre as partículas. As condições para a existência de um comportamento macroscópico são asseguradas quando o espalhamento intrapartícula sobrepõe o espalhamento interpartícula. Johnson et al. (1983) destacam as seguintes características com relação à existência do comportamento macroscópico: (a) partículas rugosas (ou seja, a escala de rugosidade é muito menor que o tamanho da partícula); (b) a escala de rugosidade é maior do que o comprimento de onda de interesse (se não ela torna-se invisível); e (c) a partícula deve possuir espessura óptica maior que o valor um.

Como a mistura macroscópica é baseada na relação linear entre os seus integrantes, houve um grande desenvolvimento de técnicas de processamento digital de imagens para a estimar a abundância nesse tipo de mistura como a Análise Linear de Mistura e seus aprimoramentos: análise do resíduo (Smith et al. 1987, Gillespie et al. 1990), análise Foreground - Background (Smith et al. 1994), análise hierárquica do Foreground - Background (Pinzón et al. 1995) e Multiple Endmember Spectral Mixture Analysis (Roberts et al. 1998).

MISTURAS MICRÓSCOPICAS OU ÍNTIMAS Na mistura íntima, os componentes são aleatoriamente distribuídos e a luz é espalhada de forma múltipla entre as espécies minerais (Singer 1981, Mustard \& Pieters 1989). Nesse tipo de mistura o comportamento espectral não é uma função linear dos seus membros puros. Vários métodos foram formulados com o propósito de estabelecer, a partir de uma mistura íntima de minerais, a proporcionalidade e abundância dos seus integrantes.

Modelos Teóricos Os modelos teóricos estão embasados nos princípios físicos de interação da energia eletromagnética com a mistura em estudo. Aproximações teóricas têm sido desenvolvidas para descrever a interação da luz com a superfície das partículas baseadas na teoria de transferência de radiatividade de Chandrasekhar (1960) (Hapke 1981 1984, 1986, Lumme \& Bowell 1981). Dentre esses modelos destaca-se o de Hapke, fundamentado na reflectância bidirecional de misturas íntimas. A formulação de Hapke tem sido muito utilizada para misturas minerais, inclusive na análise fotométrica de Mercúrio, da Lua e de outros satélites (Hapke \& Wells 1981, Mustard \& Pieters 1987).

O modelo de reflectância é função da geometria de visada onde as diferentes variáveis retratam as propriedades físicas e o comportamento de espalhamento da superfície. Contemplando essa característica, o desenvolvimento do modelo Hapke tem possibilitado várias soluções para o problema de transferência

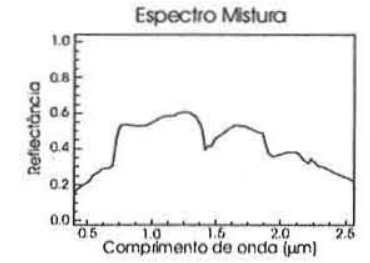

Solo
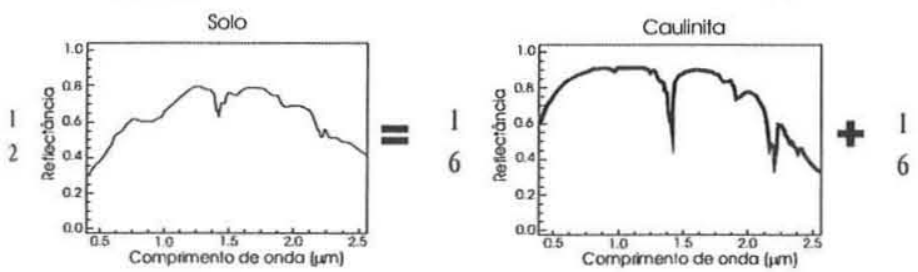

Vegetação

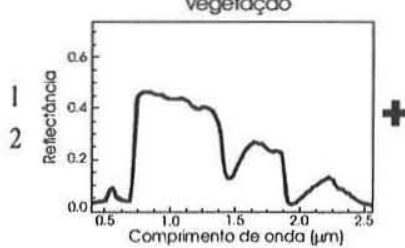

Caulinita

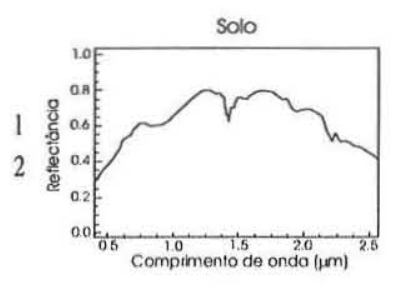

Gibsita

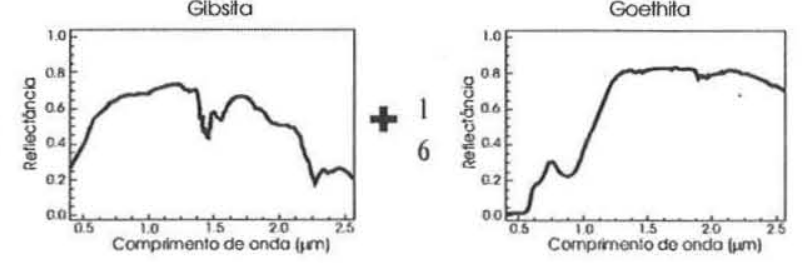

Figura 1 - Simulação de uma mistura macroscópica considerando uma célula composta de metade por vegetação e metade por solo onde este último é subdividido em três porções iguais de caulinita, gibsita e goethita. 
dentro de um meio particulado onde a diferença primária consiste na natureza da radiância reflectiva medida (i.e., reflectância direcional-hemisférica, reflectância Lambertiana, albedo normal, etc). A formulação de Hapke consta da equação (2):

$\mathrm{R}(\lambda)=\mathrm{w} /(4(\mu+\mu \mathrm{o}) *((1+\mathrm{B}(\mathrm{g})) \mathrm{P}(\mathrm{g})+\mathrm{H}(\mu) \mathrm{H}(\mu \mathrm{o})-1)(2)$ onde $R(\lambda)=$ reflectância para o comprimento de onda $\lambda, w=$ média do albedo de espalhamento simples; $\mu=\cos (\mathrm{e}),(\mathrm{e})=$ ângulo de emergência ou detecção; $\mu \mathrm{o}=\cos (\mathrm{i})$, (i) = ângulo de incidência; $\mathrm{g}$ = a fase do ângulo; $\mathrm{B}(\mathrm{g})=$ função de espalhamento; $\mathrm{P}(\mathrm{g})=$ função média da fase de partícula simples e $\mathrm{H}=$ função de Chandrasekhar para espalhamento isotrópico.

A determinação da reflectância pela derivação do albedo de um espalhamento simples usando a equação (2) requer múltiplas medições em diferentes geometrias de visada. Uma vez que a geometria múltipla de visada não é, comumente, obtida pelas técnicas atuais de espectroscopia de imageamento atuais uma alternativa é o desenvolvimento de métodos empíricos para o cálculo de abundância de misturas íntimas.

Modelos Empíricos Relacionam as propriedades mensuráveis de feições de absorção com a abundância dos membros finais. Esse tipo de modelo é específico para uma determinada mistura. Para cada mistura torna-se necessária a calibração em laboratório com uma série que abrange as diferentes proporções das assembléias minerais em estudo (Adams 1974, Singer 1981, Cloutis et al. 1986). A partir das propriedades mensuráveis do espectro estabelecem-se índices que melhor retratem o comportamento da mistura.

As medições do espectro são realizadas tanto para toda a curva espectral quanto apenas para determinadas faixas espectrais relativas às feições de absorção que caracterizam um dado mineral. Para estas medições são utilizados vários parâmetros morfométricos como dimensões de área, razões de bandas, profundidade da banda de absorção (Clark \& Roush 1984), largura da feição de absorção, assimetria, entre outros. Dentre os mais utilizados destacam-se a razão de banda e a profundidade da banda espectral também denominada de razão de três bandas. No entanto, esses parâmetros morfométricos nem sempre apresentam uma relação linear com a quantidade real do mineral em análise. Desta forma, esses índices também devem ser calibrados de acordo com a abundância real do material (Clark \& Lucey 1984).

ESPECTROMETRIADASAMOSTRASDEGARNIERITA Para a obtenção dos espectros das amostras de garnierita da mina Fazenda utilizou-se um espectroradiômetro da marca GER (Geophysical \& Environmental Research Corporation), modelo IRIS (Infrared Intelligent Spectroradiometer) MARK V. Este instrumento opera na faixa espectral de 300 a $2500 \mathrm{~nm}$, com larguras de bandas de $2 \mathrm{~nm}$ no intervalo de 300 a $1000 \mathrm{~nm}$ e de $4 \mathrm{~nm}$, entre 1000 a $2500 \mathrm{~nm}$.

O termo garnierita define uma mistura de hidrossilicatos de magnésio e níquel (Trecasses et al. 1980, Brindley \& Pham 1973). A garnierita da mina Fazenda é predominantemente constituída por pimelita e saponita. Sua cor varia de verde, na base do perfil, até marrom, no topo, devido à entrada de goethita.

O emprego da espectrorradiometria na análise mineralógica de garnierita vem sendo alvo de estudos. Maquet et al. (1981) identificaram nas garnieritas da Nova Caledônia, mistura de serpentina e tipos intermediários de talco e saponita, feições espectrais de transições eletrônicas de campo cristalino, decorrentes da presença do íon $\mathrm{Ni2}+$ em coordenação octaédrica, em $0,39 \mu \mathrm{m}, 0,66 \mu \mathrm{m}$ e $1,100 \mu \mathrm{m}$. Cervelle \& Maquet (1982) apresentaram uma leve mudança das duas primeiras feições para garnieritas, passando a considerar os valores de $0,38 \mu \mathrm{m} \mathrm{e} 0,67 \mu \mathrm{m}$. Porto (1994) identificou dois tipos de garnieritas para o complexo máfico - ultramáfico de Barro Alto: uma com predomínio de serpentina e a outra de talco. O comportamento espectral dessas garnieritas apresentava as feições do $\mathrm{Fe}$ e do $\mathrm{Ni}$ descritas por Maquet et al. (1981).

A garnierita da área de estudo diferencia-se por ser constituída principalmente por pimelita, apresentando feições de transição

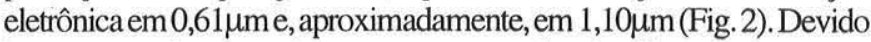
à presença de magnésio na estrutura da pimelita observam-se feições devido às vibrações na ligação $\mathrm{Mg}-\mathrm{OH}$ em $2,29 \mu \mathrm{m}$ e $2,385 \mu \mathrm{m}$. As feições relativas às bandas de água $1,4 \mu \mathrm{m}$ e $1,9 \mu \mathrm{m}$ também se apresentam bem demarcadas no espectro.

Com o aumento da proporção de goethita observa-se um deslocamento das feições espectrais da garnierita na faixa do visível. Sherman \& Waite (1985) descrevem as feições de transições eletrônicas de campo cristalino do $\mathrm{Fe}^{+3}$ para as goethitas em $0,435 \mu \mathrm{m}, 0,480 \mu \mathrm{m}, 0,650 \mu \mathrm{m}, 0,917 \mu \mathrm{m}$. Para demonstrar essa mudança do comportamento espectral gerou-se uma série de espectros entre a pimelita e goethita (Fig. 3).

As principais características do comportamento espectral das amostras de garnierita com o aumento do $\mathrm{Fe}^{+3}$ são: (a) deslocamento das feições de absorção de transição eletrônica do $\mathrm{Ni}^{+2}$ para oFe ${ }^{+3}(0,38 \mu$ m para $0,46 \mu \mathrm{m} ; 0,61 \mu$ m para $0,66 \mu \mathrm{m} ; \mathrm{e} 1,10 \mu \mathrm{m}$ para $0,925 \mu \mathrm{m})$; (b) a feição relativa à ligação $\mathrm{Mg}-\mathrm{OH}$ mantém-se até o completo predomínio da goethita; $\mathrm{e}$ (c) diminuição gradativa da feição da absorção $\mathrm{H}_{2} \mathrm{O}$ com o aumento da goethita.

Outras mudanças que podem ocorrer com os espectros da garnierita são relativas à presença de minerais como a saponita, antigorita e asbolana. A quantidade de saponita aumenta à medida que aumenta a goethita. As principais feições para a distinção da pimelita e saponita estão localizadas na região do visível. No entanto, para a mina Fazenda, devido à presença da goethita, ocorre uma obliteração dessas feições. Uma outra distinção entre esses dois minerais pode ser feita através das feições vibracionais da ligação

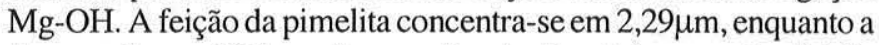
da saponita, em 2,31 $\mu \mathrm{m}$. A serpentina (antigorita) apresenta a feição da ligação $\mathrm{Mg}-\mathrm{OH}$ em 2,32 $\mu \mathrm{m}$. Além disso, apresenta uma outra

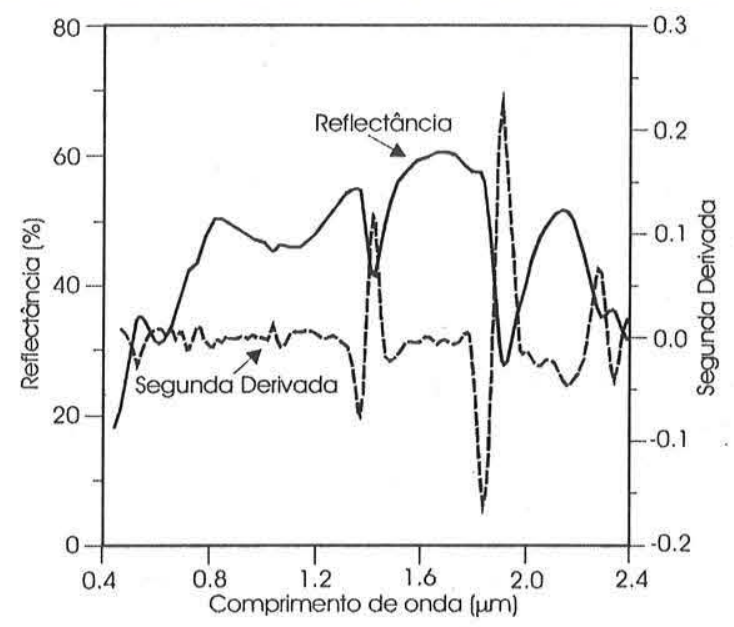

Figura 2 - Espectro da reflectância da garnierita pimelítica e a sua segunda derivada realçando os pontos de absorção. 
feição vibracional em $2,11 \mu \mathrm{m}$, o que permite sua distinção da pimelita-saponita. A asbolana é um mineral opaco caracterizado por uma banda de condução onde os elétrons possuem muito mais energia do que os de valência, pois estão livres para moverse na malha cristalina. O comportamento desses elétrons livres ou de condução provoca uma forte absorção ao longo do espectro.

Para o presente trabalho é analisada a mistura entre os dois principais minerais presentes no perfil de intemperismo da mina Fazenda: a pimelita e a goethita. Para enfocar o comportamento da mistura íntima também foi abordado o comportamento relativo entre a pimelita e a asbolana.

Para a análise de mistura espectral foram realizadas simulações tanto de mistura macroscópica como de misturas íntimas, gerando uma série contínua entre os componentes minerais. As misturas macroscópicas foram simuladas utilizando proporções lineares. As misturas íntimas foram simuladas em laboratório a partir da mistura do pó dos minerais seguida da medição radiométrica. As amostras, que já apresentavam bastantes friáveis, foram destorroadas, moídas e peneiradas para obter partículas menores que 200 mesh. As porcentagens entre minerais foram determinadas por relação volumétrica. No entanto, deve-se destacar que na mistura íntima existem vários condicionantes que alteram o seu comportamento final como a granulometria e a rugosidade. Além disso, na natureza os minerais podem ocorrer de forma diferenciada que pode mascarar o resultado final do espectro como, por exemplo, as feições do tipo red beds, onde os grãos de quartzo são envolvidos por uma camada de goethita.

O presente trabalho considera em condições laboratoriais o comportamento da mistura espectral tanto macroscópica como íntima de forma a estabelecer as condições envolventes entre as duas eondições. Para os modelos de mistura foram calculados os seguintes índices: coeficientes angulares e lineares relativos à regressão linear entre o espectro padrão e o espectro da mistura (Harsanyi \& Chang 1994) e o coeficiente escala (Clark \& Roush 1984). Com a finalidade de avaliar as interferências provenientes da similaridade entre os espectros puros calculou-se também o coeficiente de correlação.

\section{ANÁLISE DA ABUNDÂNCIA DA GARNIERITA QUANDO}

MISTURADA COM GOETHITA Na mina Fazenda o perfil de intemperismo apresenta uma mistura da pimelita com a goethita, tanto na forma macroscópica como microscópica. Para o cálculo de abundância da pimelita utilizou-se a feição centrada em 2,29 $\mu \mathrm{m}$, referente à ligação $\mathrm{Mg}-\mathrm{OH}$. Nessa feição existe pouca influência dos demais minerais presentes no perfil laterítico (Fig. 3).

A série de mistura macroscópica é apresenta na figura $4 \mathrm{em}$ intervalos de $10 \%$ de abundância em volume. O comportamento dos coeficientes angular e linear em relação à abundância de pimelita são estabelecidos segundo uma função linear (Fig. 5). No entanto, observa-se que o coeficiente angular não atinge o valor zero na inexistência da pimelita. Isto é comum e demonstra uma interferência proveniente de uma leve curvatura nessa porção do espectro da goethita que gera baixos valores dos coeficientes mesmo na inexistência de pimelita. Esse comportamento dificulta o cálculo de abundância para baixas porcentagens de pimelita que são susceptíveis a esse tipo de interferência. Outra característica que acentua a presença da interferência é a correlação alta entre os espectros puros, que atinge valores de 0,90 (Fig. 6). Devido a essas interferências torna-se fundamental uma calibração prévia dos dados para ajustar os valores dos coeficientes com o início real da presença do elemento procurado.
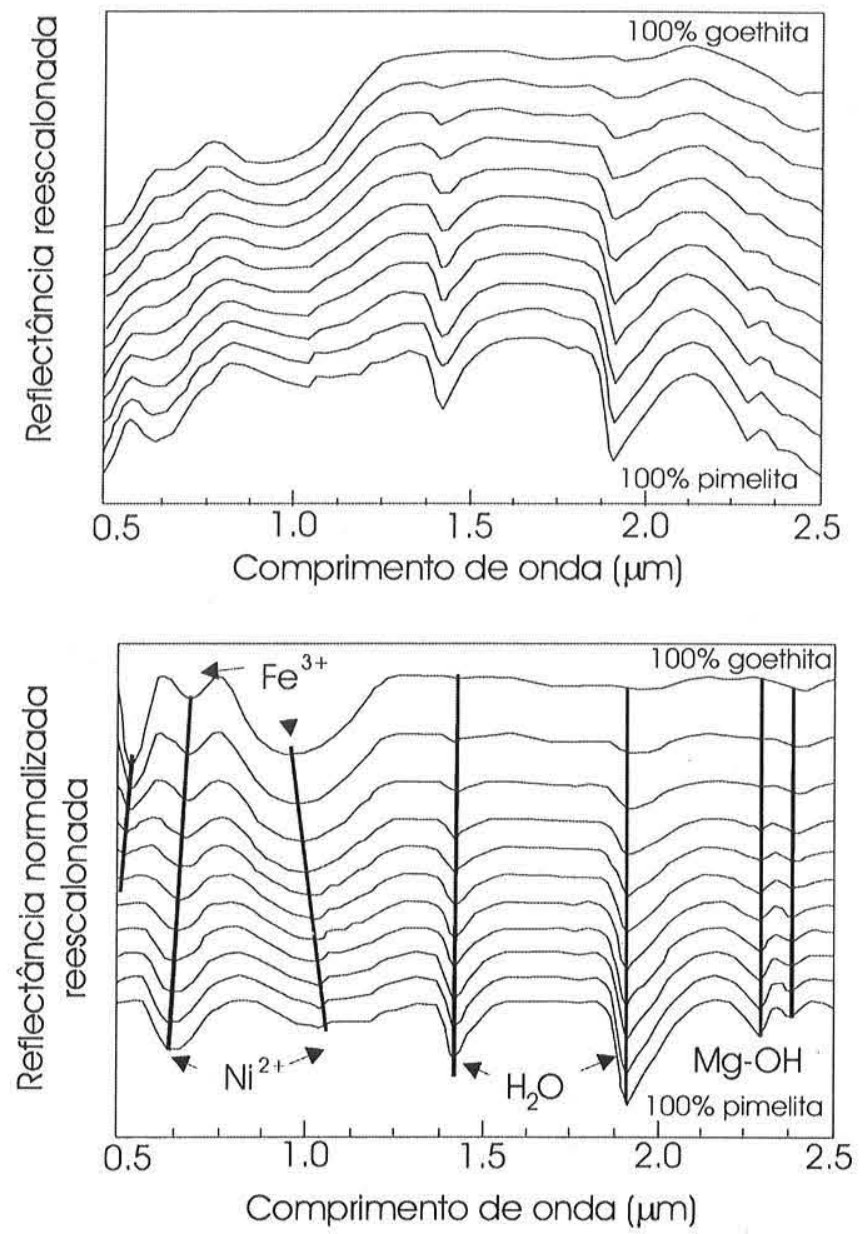

Figura 3 - Comportamento espectral de uma série de misturas entre pimelita e goethita sem e com normalização (remoção do contínuo) obtidas por simulação linear. A abundância relativa em volume entre a pimelita e goethita varia inversamente de 0 a $100 \%$ em intervalos de $10 \%$. Na figura da direita as barras sinalizam o deslocamento da banda central de absorção demonstrando sua importância na identificação mineral.

O coeficiente escala não é tão afetado pela correlação dos espectros, com valores próximos de zero na ausência da pimelita. No entanto, observa-se na figura 5 que o coeficiente escala não apresenta um comportamento linear com a abundância mineral sendo necessário o estabelecimento de uma função de ajuste não linear para o seu emprego preciso na quantificação da pimelita.

Para a série de misturas íntimas entre a goethita e a pimelita realizadas em laboratório foram empregados também intervalos de $10 \%$ de abundância. O comportamento da banda de absorção da pimelita em diferentes proporções de mistura com a goethita é apresentado na figura 7. Observa-se que o coeficiente de correlação na ausência da pimelita apresenta também valores altos demonstrando uma alta correlação entre os espectros (Fig. 8). Os coeficientes calculados em relação à abundância apresentam-se dispostos segundo funções lineares e coeficientes de correlação da regressão superiores que $99 \%$ de ajuste (Figs. 9, 10 e 11). Desta forma atesta-se que o emprego de qualquer um dos parâmetros utilizados para quantificação é eficaz para estimar a proporção 
mineral

ANÁLISE DA ABUNDÂNCIA DA PIMELITA MISTURADA COM ASBOLANA A fácies garnierítica contém veios de asbolana. A análise dessa mistura é interessante para demonstrar o comportamento das misturas espectrais quando existe uma forte variação do albedo.

A figura 12 apresenta a série de misturas macroscópica entre a pimelita e a asbolana. $O$ coeficiente angular estabelece uma perfeita relação linear expressando a porcentagem da pimelita na amostra (Fig. 13). O coeficiente escala, como esperado, não fornece o mesmo resultado. A feição retilínea da asbolana para a faixa do espectro analisada proporciona baixo valor de correlação entre os membros finais o que favorece a análise (Fig. 14).

Na mistura íntima observa-se uma intensa queda na reflectância da pimelita devido à presença da asbolana (Fig. 15). Esses resultados são consistentes com estudos prévios de mistura de assembléias minerais de opacos com não opacos (Nash \& Conel
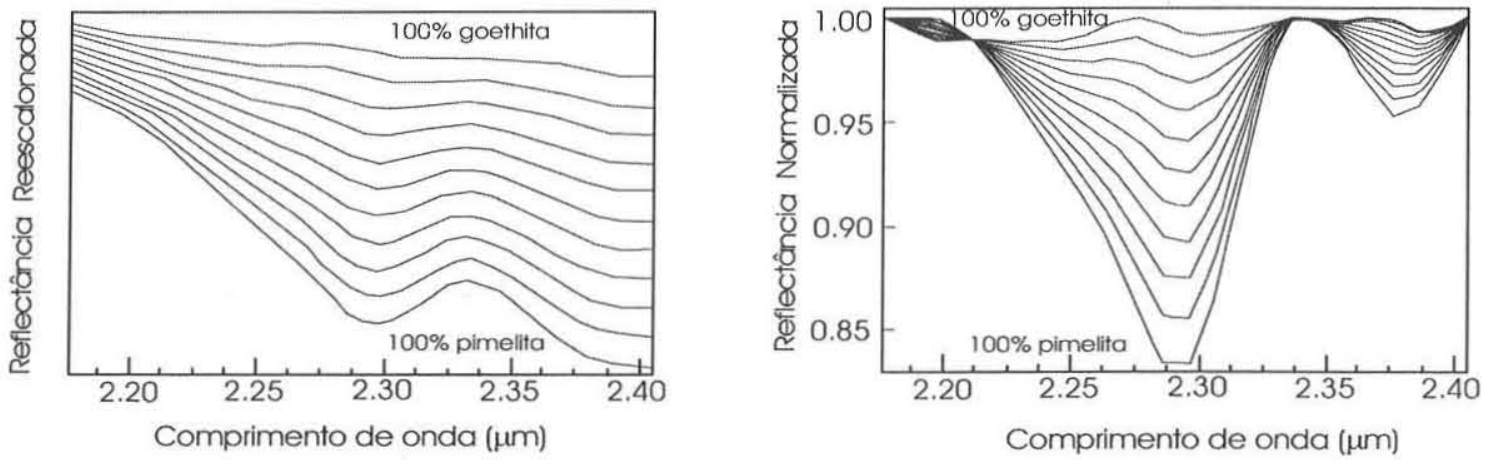

Figura 4 - Simulação das misturas macroscópicas entre pimelita e goethita com intervalo de abundância de 10\%: (a) sem normalização (remoção do contínuo) e (b) com normalização.

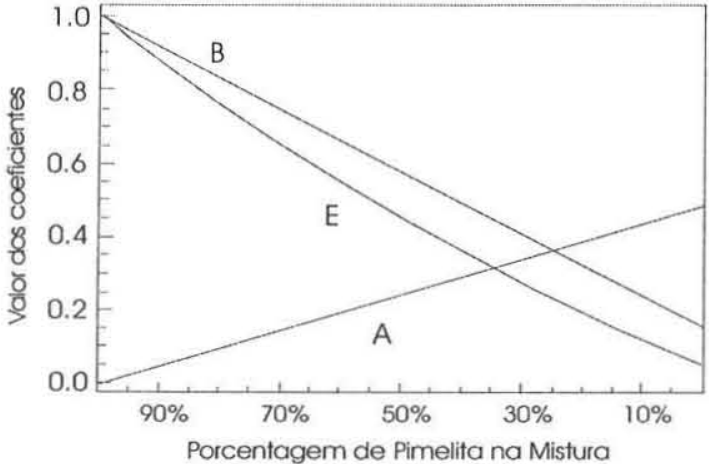

Figura 5 - Comportamento do coeficiente angular (B), linear (A) e escala (E) para os modelos de misturas macroscópicas entre a pimelita e a goethita.

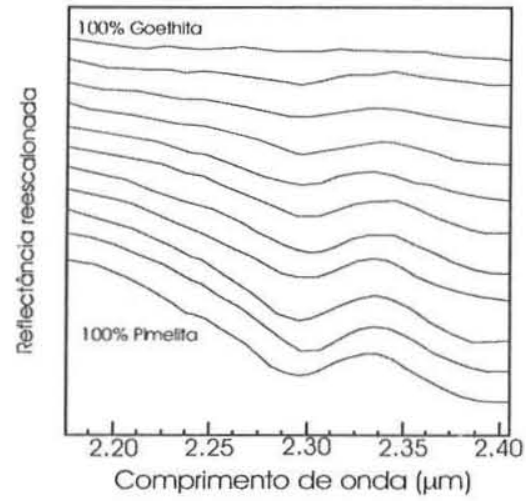

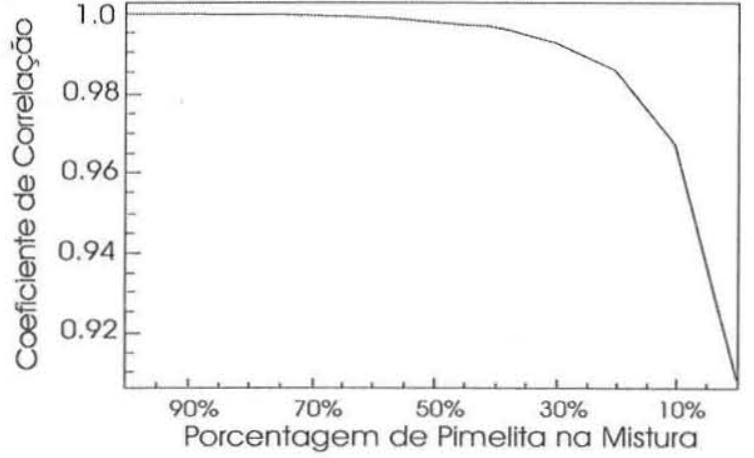

Figura 6 - Coeficiente de correlação entre a curva de pimelita pura e as demais curvas de mistura macroscópica entre a pimelita e de goethita.

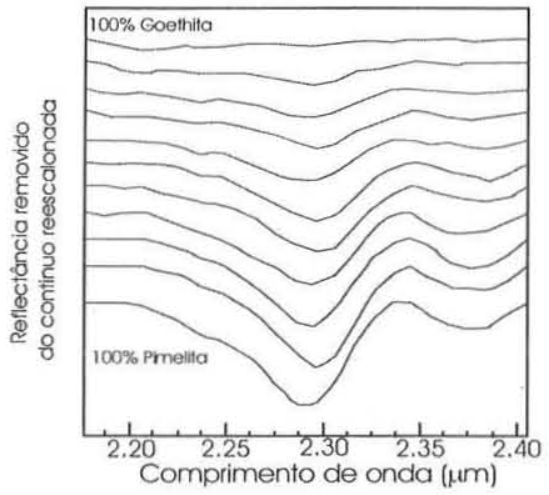

Figura 7 - Simulação de mistura íntima entre pimelita e goethita com intervalo de abundância entre as curvas de 10\%: a) sem normalização (remoção do contínuo) e b) com normalização. 
1974, Madeira 1991). Os coeficientes angular e escala em relação à abundância de pimelita estão dispostos segundo uma função logarítmica (Figs. 16 e 17). Nesse caso, o coeficiente escala não proporcionou uma disposição linear dos dados, demonstrando a importância do balizamento com dados experimentais. A função logarítmica determinada pode ser aplicada sobre os dados proporcionando uma melhor estimativa da quantidade da pimelita para áreas com mistura com asbolana.

CONCLUSÃO Como cada mistura tem comportamento

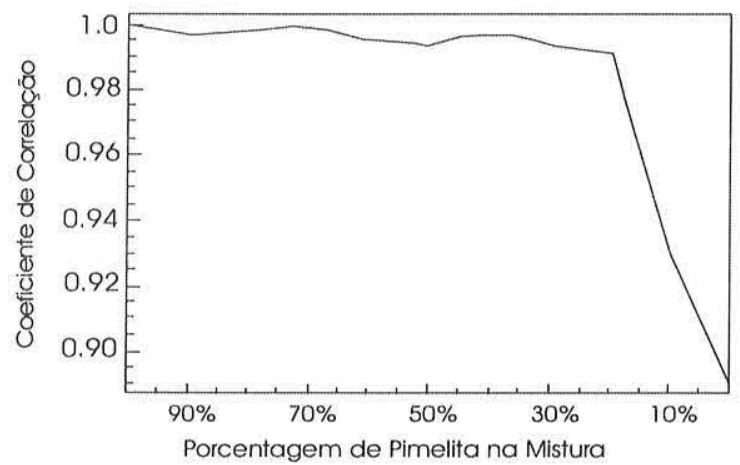

Figura 8 - Valores de coeficiente de correlação entre pimelita e as curvas relativas à mistura com goethita.

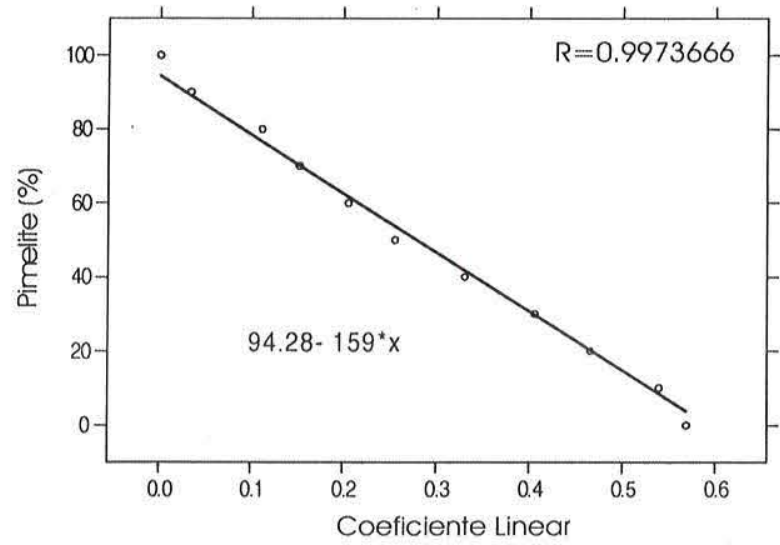

Figura 9 - Comportamento do coeficiente linear para a mistura intima entre a pimelita $e$ a goethita apresentando uma distribuição linear.

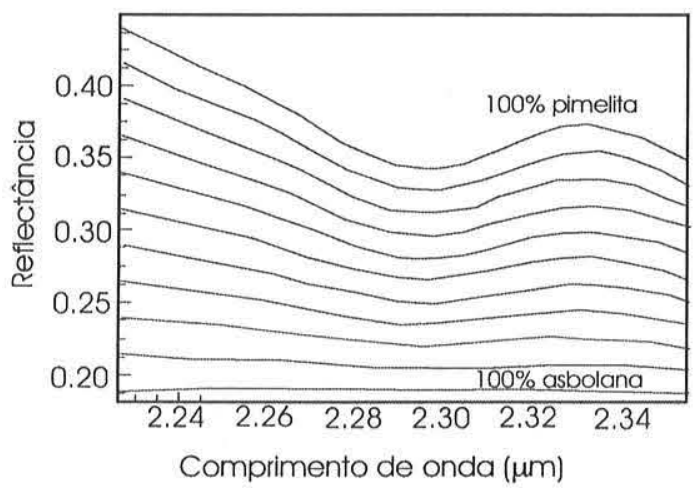

específico, o emprego de simulações permite verificar e corrigir equívocos inerentes aos métodos de determinação de abundância. Observa-se que os índices morfométricos fornecem uma escala de valores que devem ser balizados por dados experimentais tanto na mistura íntima como na mistura macroscópica.

Na mistura macroscópica o coeficiente angular da regressão linear entre o espectro padrão e o espectro da mistura pode apresentar superestimativas na correlação entre os espectros. Nos experimentos realizados, a abundância da pimelita na mistura com

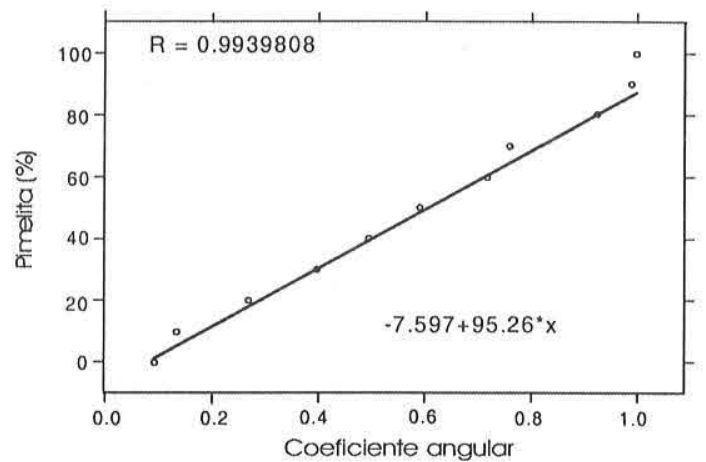

Figura 10 - Comportamento do coeficiente angular para a mistura intima entre pimelita e goethita.

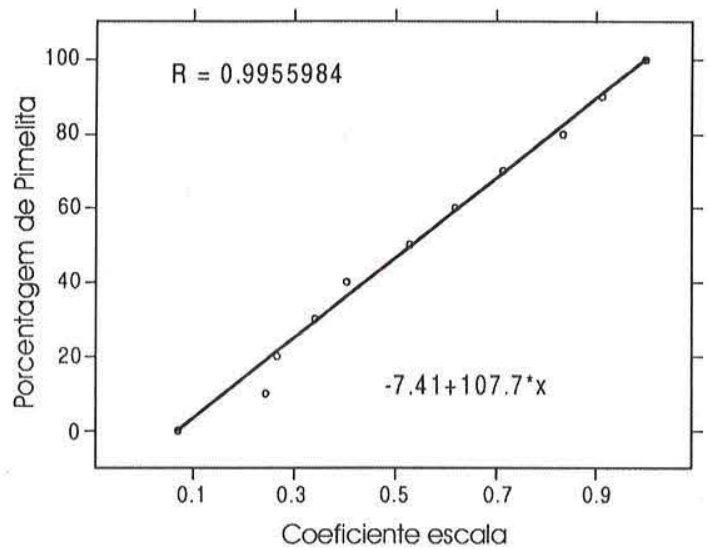

Figura 11 - Comportamento do coeficiente escala para a mistura intima entre a pimelita e a goethita apresentando uma distribuição linear:

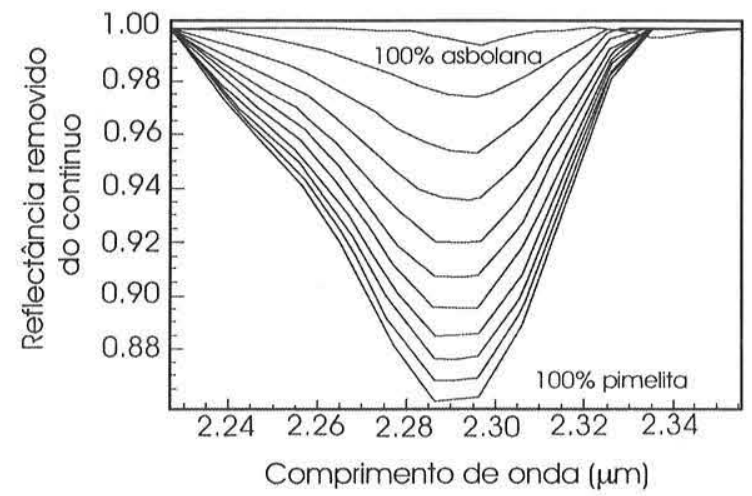

Figura 12 - Simulação da mistura macroscópica entre a pimelita e a asbolana, com intervalo de 10\%: (a) sem normalização (remoção do contínuo) e (b) com normalização. 


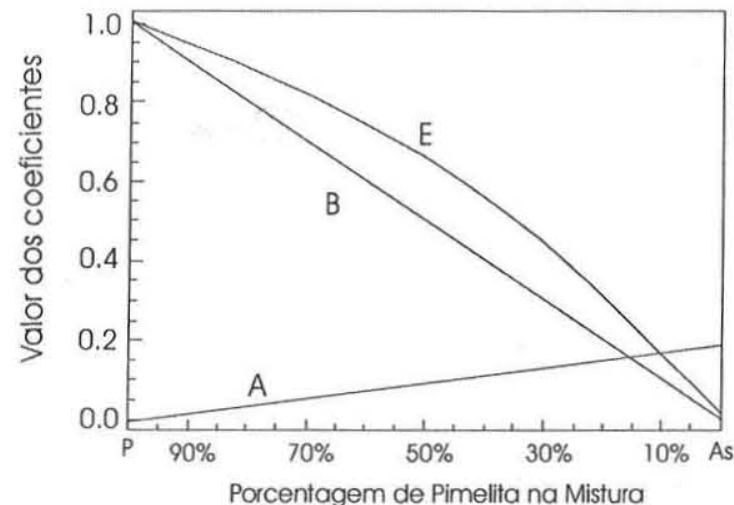

Figura 13 - Coeficiente angular (B), linear (A) e escala (E) das misturas macroscópicas entre pimelita e asbolana.

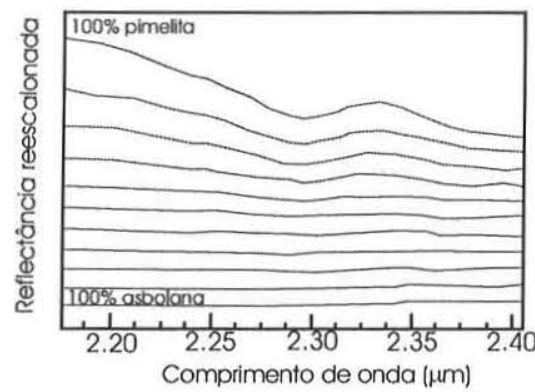

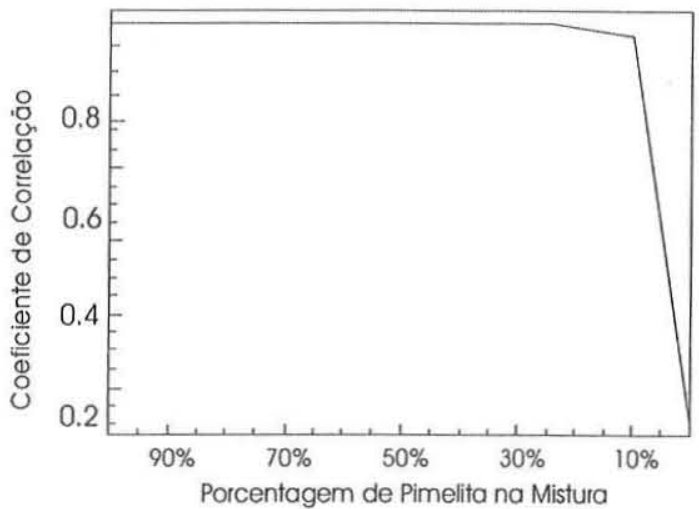

Figura 14 - Coeficiente de correlação entre a pimelita e as curvas relativas às misturas macroscópicas entre a pimelita e a goethita.

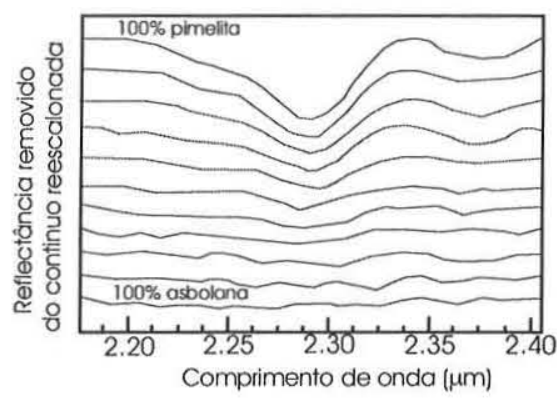

Figura 15 - Simulação de mistura íntima da pimelita e asbolana em intervalo de 10\%: (a) sem normalização (remoção do contínuo) $e$ (b) com normalização.

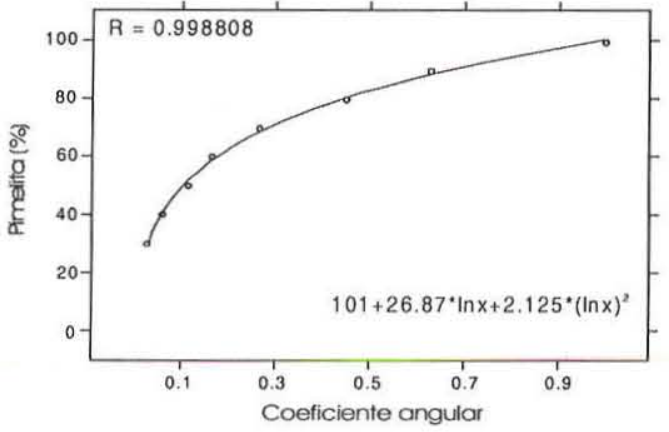

Figura 16 - Comportamento logarítmico do coeficiente angular para a mistura íntima entre a pimelita e a asbolana.

goethita, através do coeficiente angular, ficou prejudicada devido a essa correlação. Na mistura íntima os índices morfométricos mostram-se também vulneráveis devendo ser ajustadas com dados experimentais, principalmente quando existe forte diferença de albedo. O emprego da função logarítmica apresentou excelentes resultados para a calibração desse tipo de mistura. $\mathrm{O}$ método proposto permite um ajuste matemático adequado dos coeficientes com a quantidade relativa dos minerais. Desta forma, os procedimentos sugeridos aumentam a acurácia da estimativa de abundância mineral da pimelita em relação a goethita e asbolana.

Outras simulações para mistura íntima, variando a granulometria e rugosidade, também são necessárias para um melhor controle do

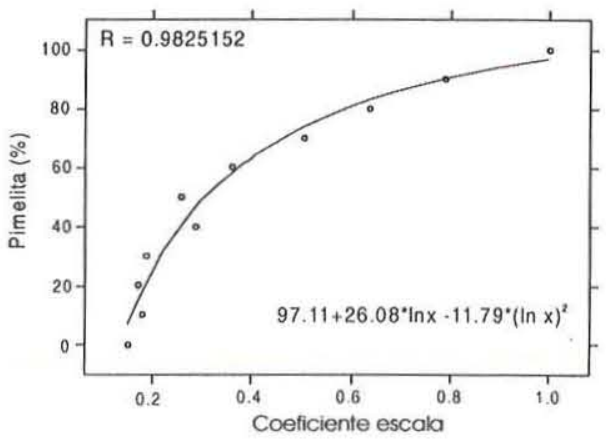

Figura 17 - Comportamento logarítmico do coeficiente escala para a mistura íntima de pimelita e asbolana.

processo. No entanto, a partir desse experimento pode-se observar os comportamentos limítrofes entre os tipos de misturas presentes na natureza. A quantificação de mistura íntima na espectroscopia de imageamento ainda apresenta muitas dificuldades, constituindo um vasto campo para pesquisa. Portanto, uma calibração desse tipo pode trazer resultados mais fidedignos. Além disso, para estudos mineralógicos rápidos junto às bancadas de mineração o emprego de espectroradiômetros de campo pode agilizar e auxiliar na priorização de análises de laboratório.

Agradecimentos Aos relatores da RBG pelas sugestões ao manuscrito. 


\section{Referências}

Adams J.B. 1974. Visible and near-infrared diffuse reflectance: spectra of pyroxenes as applied to remote sensing of solid objects in the solar system. J. Geophys. Res., 79:4829-4836.

Brindley G.W. \& Pham T.H. 1973. The nature of garnierites: I - structure chemical composition and color characteristics. Clays Clay Min.; 21:17-40.

Carvalho Júnior O.A. 2000. Avaliação e desenvolvimento de métodos de processamento de imagens hiperespectrais - análise em depósito de níquel (Niquelândia, GO). Tese de doutorado, UnB, 262p.

Cervelle B.D. \& Maquet M. 1982. Cristallochimie des lizardites substituees $\mathrm{Mg}$ - $\mathrm{Fe} \_\mathrm{Ni}$ par spectrometrie visible et infrarouge proche. Clay Min., 17:377-392.

Clark R.N. \& Lucey P.G. 1984. Spectral properties of ice-particulate mixture and implications for remote sensing 1 . intimes mixtures. $J$. Geophys. Res., 89:6341-6348

Clark R.N. \& Roush T.L. 1984. Reflectande spectroscopy: quantitavive analysis techniques for remote sensing applications. J. Geophys. Res., 89:6329-6340.

Cloutis E.A., Gaffey M.J., Jackowski T.L., Reed K.L. 1986. Calibrations of phase abundance, composition, and particle size distribution for olivine-orthopyroxene mixtures from reflectance spectra. J. Geophys. Res., 91: B11:11641-11653.

Colin F., Nahon D., Trescases J.J., Melfi A.J. 1990. Lateritic weathering of pyroxenites at Niquelandia, Góias, Brazil: the supergene behavior of Nickel. Econ. Geol., 85: 1010-1023.

Colin F., Noack Y., Trecasses J.J., Nahon D. 1985. L'altération latéritique débutante des pyroxénites de Jacuba, Niquelândia, Brésil. Clay Minerals, 20:93-113.

Gillespie A.R., Smith M.O., Adams J.B., Willis S.C., Fischer A.F., Sabol D.E. 1990. Interpretation of residual images: spectral mixture analysis of AVIRIS images, Owens Valley, California. In: Annual JPL Airborne Visible/Infrared Imaging Spectrometer (AVIRIS) Workshop, 2, Pasadena, CA, Summaries, JPL Publ. 90-54, 243-270.

Hapke B. \& Wells E. 1981. Bidirectional reflectance spectroscopy: 2 experiments and observations. J. Geophys. Res., 86:3055-3060.

Hapke B. 1981. Bidirectional reflectance spectroscopy, 1, Theory. J. Geophys. Res., 86:3039-3054.

Hapke B. 1984. Bidirectional reflectance spectroscopy, 3, Correction for macroscopic roughness. Icarus, 59:41-59.

Hapke B. 1986. Bidirectional reflectance spectroscopy, 4, The extinction coefficient and the opposition effect. Icarus, 67:264-287.

Harsanyi Chang. 1994. Hyperspectral image classification and dimensionality reduction: an orthogonal subspace projection approach. IEEE Trans. Geosci. and Remote Sens., 32:779-785.

Johnson P.E., Smith M.O., Taylor-George S. \& Adams J.B. 1983. A semi-empirical method for analysis of the reflectance spectra of binary mineral mixtures. J. Geophys. Res., 88:3557-3561.

Jurkovic I. 1963. Some geochemical aspects about the genesis of the nickel deposit Loma de Hierro (Venezuela). Geolos. Vjesnik. Zaghreb$Y U$., 17:103-112.

Lumme K. \& Bowell E. 1981. Radiative transfer in the surfaces of atmospheres bodies, I: Theory. Astron. J., 86:1694-1704.

Madeira Netto J.S. 1991. Étude quantitative des relations constituants mineralogiques-réflectance difuse des latosols brésiliens/application al'utilisation pedologique des données satellitaires TM (regionde Brasília). Thése de Doctorat, Université Pierre et Marie Curie. Paris. 224 p.
Maquet M., Cervelle B.D., Gouet G. 1981. Signatures of $\mathrm{Ni}^{2+}$ and $\mathrm{Fe}^{3+}$ in the optical spectra of limonitic ore from New Caledonia: Application to the determination of the nickel content. Mineral. Depos., 16:357-373.

Melfi A.J., Trescases J.J., Oliveira S.M.B. 1980. Les "Latérites" nickélifères du Brésil. Cah. O.R.S.T.O.M. Sér: Géol., 11, 1:15-42.

Mustard J.F. \& Pieters C.M. 1987. Abundance and distribution of ultramafic microbreccia in Moses Rock dike: quantitative application mapping spectroscopy. J. Geophys. Res., 92:10376-10390.

Mustard J.F. \& Pieters C.M. 1989. Photometric phase functions of common geologic minerals and applications to quantitative analysis of mineral mixture reflectance spectra. J. Geophys. Res., 94, B10:13.619-13.634.

Nash D.B. \& Conel J.E. 1974. Spectral reflectance systematics for mixtures of powdered hypersthene, labradorite and ilmenite. J. Geophys. Res., 79:1615-1621.

Oliveira S.M.B. \& Trescases J.J. 1980. Geoquímica de alteração supergena das rochas ultramáficas de Santa Fé (Goiás, Brasil), Rev. Bras. Geoc., 10:243-257.

Pinzón J.E., Ustin S.L., Hart Q.J., Jacquemoud S., Smith M.O. 1995. Using foreground/background analysis to determine leaf and canopy chemistry. In: Annual JPL Airborne Visible/Infrared Imaging Spectrometer (AVIRIS) Workshop, 5, Pasadena, CA, Summaries, JPL Publ. 95-1, 129-132.

Porto S.G. 1994. Comportamento espectral das lateritas niquelíferas do complexo máfico-ultramáfico de Barro Alto, Goiás. Disertação de Mestrado, IG-UFRGS, $196 \mathrm{p}$.

Roberts D.A., Gardner M., Church R., Ustin S., Scheer G., Green R.O 1998. Mapping chaparral in the Santa Monica Mountains using multiple endmember spectral mixture models. Remote Sens. Environ., 65:267-279.

Santos-Ynigo L. 1964. Distribution of iron, alumina and silica in the Pujada laterite of Mati, Davao Province, Mindanao Island (Philippines). In: Intern. Geol. Congr., 22, New Dehli., 14:126-141.

Singer R.B. 1981. Near-infrared reflectance of mineral mixtures: Systematic combinations of pyroxenes, olivine, and iron oxides. J. Geophys. Res., 86:7967-7982.

Smith M.O., Roberts A.D., Hill J., Mehl W., Hosgood B., Verdebout J., Schmuck G., Koechler C., Adams J. 1994. A new approach to quantifying abundances of materials in multispectral images. In: IGARSS 1994, Proceedings, 2372-2674.

Smith M.O., Roberts D.A., Shipman H.M., Adams J.B., Wills S.C., Gillespie A.R. 1987. Calibrating AIS images using the surface as reference. In: Airborne Imaging Spectrometer Workshop, 3, Proceedings, JPL Publ., 87-30, 10p.

Trescasses J.J., Melfi A.J., Oliveira S.M.B., 1980, Nickeliferous laterites of Brazil. In: Intern. Seminar Laterisation Process. Trivandrum, Índia, Proceeding, 170-184.

Turner R.A. 1968. The distribution and association of nickel in the ferruginous zones of laterites of the Giles complex, Australia. Australian Mineral Devel. Lab. Bull., 5:76-93.

Waal S.A. de. 1971. South African nickeliferous serpentinites. Min. Sci. Eng., 3:32-45.

Manuscrito A-1455

Recebido em 05 de agosto de 2003

Revisão dos autores em 25 de julho de 2004 Revisão aceita em 28 de julho de 2004 